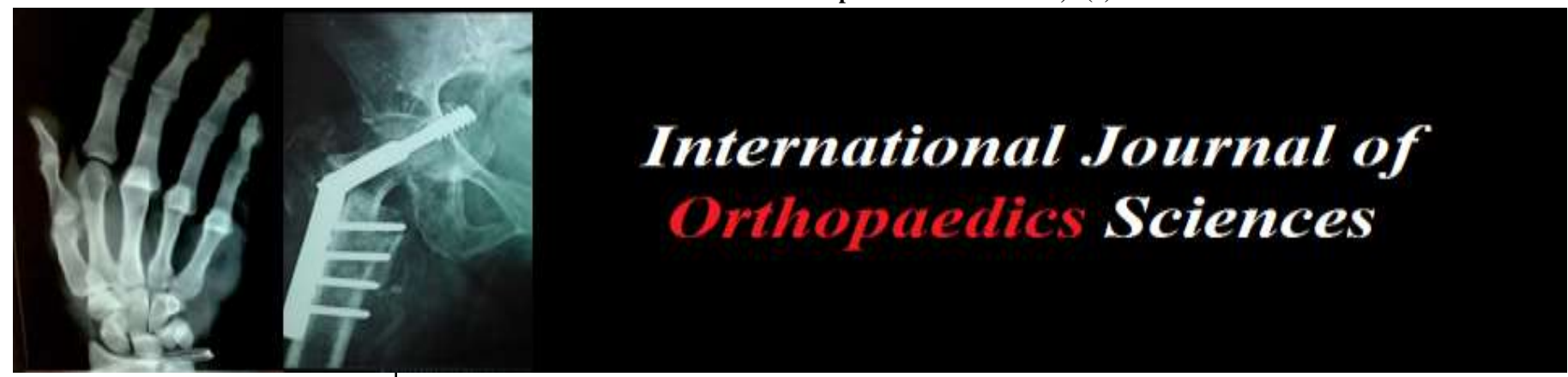

E-ISSN: 2395-1958

P-ISSN: 2706-6630

IJOS 2021; 7(1): 483-485

(C) 2021 IJOS

www.orthopaper.com

Received: 15-11-2020

Accepted: 21-12-2020

Dr. Hrishikesh Suresh Saodekar Associate Professor, Department of Orthopedics, Geetanjali Medical College and Hospital, Udaipur, Rajasthan, India

Dr. Kamal Kumar Agarwal Assistant Professor, Department of Orthopedics, Geetanjali Medical College and Hospital, Udaipur, Rajasthan, India
Corresponding Author: Dr. Kamal Kumar Agarwal Assistant Professor, Department of Orthopedics, Geetanjali Medical College and Hospital, Udaipur, Rajasthan, India

\section{Evaluation of patients with cervical spine injury and predicting the risk and severity of acute spinal cord injury after a minor trauma}

\section{Dr. Hrishikesh Suresh Saodekar and Dr. Kamal Kumar Agarwal}

DOI: https://doi.org/10.22271/ortho.2021.v7.i1h.2530

Abstract

Introduction: The relevance of MR image parameters for predicting the risk and severity of acute SCI in patients after a minor trauma to the cervical spine has not yet been established. The aim of the present study was to investigate the spinal canal diameter and the space available for the cord at the level of the intervertebral disc in patients suffering from acute cervical SCI after a minor trauma to the cervical spine. Materials \& Methods: On the magnetic resonance images the following parameters were recorded: sagittal diameter of the vertebral body, the sagittal outer diameter of the subarachnoid space at the midpoint of the vertebra, the sagittal outer diameter of the subarachnoid space at the level of the intervertebral disc, and the sagittal diameter of the spinal cord at the levels $\mathrm{C} 2$ and $\mathrm{T} 1$.

Results: it was found that there were significant and positive correlations between investigated parameters (i.e., mid vertebral and disc-level canal diameters, TPR-MRI). No significant correlations between any of the parameters and height or weight were detected. Furthermore, there was no significant correlation between any of the parameters and the ASIA motor or sensory score.

Conclusions: Assessment of the number of segments of the spinal cord involved, by MR Imaging is helpful in predicting the prognosis of the patient. Present study states that patient at risk of acute SCI after minor trauma can be identified by applying a disc level canal.

Keywords: Spinal injury, magnetic resonance, spinal canal diameter, disc level

\section{Introduction}

Spinal cord injury (SCI) is a debilitating neurological condition with tremendous socioeconomic impact on affected individuals and the health care system. It is well known in the literature that cervical spinal canal stenosis (CSCS) is an important risk factor for spinal cord injury (SCI) after trauma ${ }^{[1,2]}$. Traumatic SCI in adults with CSCS is usually seen in elderly patients, and it may be seen even after minor trauma. In most countries, acute spinal cord injury occurs at an annual rate of $20-40$ persons per million. The main causes of spinal cord trauma are motor vehicle accidents, sports and recreational activities, accidents at work and falls at home ${ }^{[3,4]}$.

Traumatic cervical spinal cord injury (SCI) is frequently a result of spinal fracture and dislocation or an outcome of discoligamentous injury. Neurologic deficits after trauma to the cervical spine may develop in the absence of bony damage, and such instances often are described as SCI without radiographic abnormality or as SCI without radiologic evidence of trauma ${ }^{[5,6]}$

Only very limited clinical evaluation of cervical spinal trauma has been done with MR Imaging. Also to minimize the possibility of failure to diagnose occult injuries, it has become standard practice in many centers to perform conventional radiographic studies in all patients admitted with a history of substantial blunt trauma ${ }^{[7]}$.

Different radiographic parameters have thus been investigated as a surrogate for spinal canal stenosis to assess the risk of SCI after a minor trauma to the cervical spine. Presently, the cervical spinal canal to vertebral body ratio is often used to assess spinal canal stenosis on conventional radiographs, which still is the first-line imaging evaluation of the non-traumatic and traumatic cervical spine ${ }^{[8,9]}$.

In the study before various authors have stated that images in the MR are very reliable for prediction of course of cervical spinal neurapraxia in the patients suffering from trauma. 
However, the relevance of MR image parameters for predicting the risk and severity of acute SCI in patients after a minor trauma to the cervical spine has not yet been established. The aim of the present study was to investigate the spinal canal diameter and the space available for the cord at the level of the intervertebral disc in patients suffering from acute cervical SCI after a minor trauma to the cervical spine.

\section{Materials \& Method}

The present study is the radiological retrospective study done in the medical college and hospital. The patient diagnosed with the acute cervical SCI after trauma were included in the study. The detail case history of the admitted patients was recorded. Prior to the inclusion of the patients, they were informed about the study and informed consent was signed by each of the patients. The institute ethical committee was informed about the study and the ethical clearance certificate was obtained prior to the start of the study.

The following exclusion criteria were applied to patients who had sustained minor trauma to the cervical spine: fractures (except for isolated fractures of spinous processes and disco ligamentous injuries of the cervical spine, patients with history of cervical spinal cord disorder were excluded in the study. A total of 100 patients admitted in the hospital were included in the study. Patients who had sustained a flexionextension trauma to the cervical spine without clinical or radiological signs of cervical spinal cord pathology and who were admitted to our institution for evaluation or rehabilitation were allocated to the control group. Control group consisted of 300 patients. The youngest age of the patient in the study was 35 years and the oldest patient was of 70 years. The mean age of the patients included in the study was 50.24 years at the time of admission.

All the measurements were recorded by the observer who was unknown about the study. On the magnetic resonance images the following parameters were recorded: sagittal diameter of the vertebral body, the sagittal outer diameter of the subarachnoid space at the midpoint of the vertebra (mid vertebral canal diameter), the sagittal outer diameter of the subarachnoid space at the level of the intervertebral disc (disc-level canal diameter), and the sagittal diameter of the spinal cord at the levels $\mathrm{C} 2$ and T1. Spinal cord measurements were not taken at the affected levels to minimize measuring errors as a result of the signal changes after SCI.

Statistical analysis:

The data was recorded and tabulated. The median and confidence interval were recorded. For statistical analysis the SPSS software was used. A value of $<0.05$ was considered significant for all statistical analyses. The chi square test was used for analysis of the statistical analysis.

\section{Results}

A total of 300 patients were included in the study. The demographic distribution of the patient in the case study group and control group suggest that control group has higher age and weight, but it was not significant. This concludes that anthropometric factors have no role in SCI. The most common cause for injury to spinal cervical area was found to be fall; approximately 150 patients had the reason of fall. Next reason was road side traffic accident. (Table 1)

Mostly patients have no neurological deficits but in falls and traffic accidents, complete and partial neurological deficit was seen. TPR CR (Torg-Pavlov ratio value on conventional radiographs); TPR MRI (Torg-Pavlov ratio value on magnetic resonance images); CD DM (sagittal disc-level canal diameter) CD (MVM) sagittal mid vertebral canal diameter; PPV, positive predictive value; NPV, negative predictive value.

Table 1: Reason for sustained spinal cervical injury in both the groups

\begin{tabular}{|c|c|c|}
\hline Causes for SCI & Control group $(\mathbf{n}=\mathbf{2 0 0})$ & Case study $(\mathbf{n}=\mathbf{1 0 0})$ \\
\hline Fall & 80 & 50 \\
\hline Road traffic accident & 70 & 38 \\
\hline Diving & 28 & 4 \\
\hline Winter sports & 21 & 6 \\
\hline Flying accidents & 1 & 2 \\
\hline
\end{tabular}

Table 2: Radiological parameters in predicting SCI

\begin{tabular}{|c|c|c|}
\hline Parameter & Control group & Case group \\
\hline TPR(CR) vs. TPR (MRI) & 0.23 & 0.28 \\
\hline TPR(CR) vs. CD (DM) & 0.06 & 0.09 \\
\hline TPR(CR) vs. CD (MVM) & 0.16 & 0.10 \\
\hline TPR(MRI) vs. CD (DM) & 0.89 & 0.95 \\
\hline TPR(MRI) vs. CD (MVM) & 0.05 & 0.02 \\
\hline CD (DM) vs. CD (MVM & 0.21 & 0.08 \\
\hline
\end{tabular}

As per table 2 it was found that there were significant and positive correlations between investigated parameters (i.e., mid vertebral and disc-level canal diameters, TPR-MRI). No significant correlations between any of the parameters and height or weight were detected. Furthermore, there was no significant correlation between any of the parameters and the ASIA motor or sensory score.

\section{Discussion}

Traumatic cervical spinal cord injury (SCI) is frequently a result of spinal fracture and dislocation or an outcome of discoligamentous injury. Neurologic deficits after trauma to the cervical spine may develop in the absence of bony damage, and such instances often are described as SCI without radiographic abnormality or as SCI without radiologic evidence of trauma. Tetraplegia or paresis after a minor trauma to the cervical spine, causing no osseous or disco ligamentous injury, has been reported to occur in patients without preceding neurologic symptoms ${ }^{[5,6]}$.

Acute spinal cord injury (SCI) after a minor trauma to the cervical spine has been reported in patients without preceding neurologic symptoms. Spinal canal stenosis may be the reason for the discrepancy between the severity of the injury and that of the trauma. The spinal canal to vertebral body ratio is often used to assess canal stenosis on conventional radiographs [10, 11]. However, the ratio does not appraise soft-tissue stenosis and canal narrowing at the level of the intervertebral disc. Parameters measured on magnetic resonance (MR) images may thus be more meaningful. The relevance of MR image parameters for predicting the risk and severity of acute SCI in patients after a minor trauma to the cervical spine has not yet been established ${ }^{[12]}$.

Present prediction values for the disc-level canal diameter are superior to those reported in the literature for the TPR CR and the space available for the cord. A mid vertebral canal diameter less than $12 \mathrm{~mm}$ and $10 \mathrm{~mm}$ is 'stenotic' and a 'risk of acute SCI' respectively. All ten MR image parameters investigated in the present study were characteristic for patients suffering from acute SCI after a minor trauma to the cervical spine. The correlation between the TPR CR and the TPR MRI was moderate, but the correlations with the other MRI parameters were weak. Other authors have reported similar correlations between the TPR CR and the TPR MRI or 
among the other MR image parameters. Age, weight, and height of the patients had no or only a negligible effect on the investigated parameters in the present study. The analysis of the disc-level canal diameter measured on MR images entailed a false negative and positive rate of $20 \%$ and $5 \%$, respectively, and the proportion of true positive test results was $84 \%$.

\section{Conclusion}

MR can be employed in acute cervical trauma to demonstrate fracture-dislocations as well as to delineate soft tissue pathologic states. Assessment of the number of segments of the spinal cord involved, by MR Imaging is helpful in predicting the prognosis of the patient. Present study states that patient at risk of acute SCI after minor trauma can be identified by applying a disc level canal.

\section{References}

1. Goldstein ML, Morewitz SJ. Chronic disorders in children and adolescents: Springer Science \& Business Media 2011.

2. Leone JE. Concepts in male health: Perspectives across the lifespan: John Wiley \& Sons 2012.

3. US LT, Query SN. Treatments for Traumatic Brain Injury with Emphasis on Transcranial Near-Infrared Laser Phototherapy.

4. Arul K, Ge L, Ikpeze T, Baldwin A, Mesfin A. Traumatic spinal cord injuries in geriatric population: etiology, management, and complications. Journal of Spine Surgery 2019;5:38.

5. Izzo R, Popolizio T, Balzano RF, Pennelli AM, Simeone A, Muto M. Imaging of cervical spine traumas. European Journal of Radiology 2019;117:75-88.

6. Dalbayrak S, Yaman O, Yilmaz T. Current and future surgery strategies for spinal cord injuries. World journal of orthopedics 2015;6:34.

7. Khandelwal S, Sharma G, Saxena U, Sakhi P, Gopal S, Saxena P. Prospective evaluation of cervical spine injuries by MRI and assessing role of MR findings in predicting prognosis. Indian Journal of Radiology and Imaging 2004;14:71.

8. Solanki GA, Alden TD, Burton BK, Giugliani R, Horovitz $\mathrm{DD}$, Jones SA et al. A multinational, multidisciplinary consensus for the diagnosis and management of spinal cord compression among patients with mucopolysaccharidosis VI. Molecular genetics and metabolism 2012;107:15-24.

9. Rizzolo SJ, Vaccaro AR, Cotler JM. Cervical Spine Trauma. Spine 1994;19:2288-98.

10. Koyanagi I, Iwasaki Y, Hida K, Akino M, Imamura H, Abe H. Acute cervical cord injury without fracture or dislocation of the spinal column. Journal of Neurosurgery: Spine 2000;93:15-20.

11. Kwon SY, Shin JJ, Lee JH, Cho WH. Prognostic factors for surgical outcome in spinal cord injury associated with ossification of the posterior longitudinal ligament (OPLL). Journal of Orthopaedic Surgery and Research 2015;10:94.

12. Aebli N, Rüegg TB, Wicki AG, Petrou N, Krebs J. Predicting the risk and severity of acute spinal cord injury after a minor trauma to the cervical spine. The Spine Journal 2013;13:597-604. 